\title{
Robust operation of microgrid energy system under uncertainties and demand response program
}

\author{
Sahar Seyyedeh Barhagh', Amin Mohammadpour Shotorbani ${ }^{2}$, Behnam Mohammadi-Ivatloo ${ }^{3}$, \\ Kazem Zare ${ }^{4}$, Ali Farzamnia ${ }^{5}$ \\ ${ }^{1,3,4} \mathrm{Faculty}$ of Electrical and Computer Engineering, University of Tabriz, Iran \\ ${ }^{2}$ School of Engineering, University of British Columbia, Canada \\ ${ }^{5}$ Faculty of Engineering, Universiti Malaysia Sabah, Malaysia
}

\begin{tabular}{l}
\hline \hline Article Info \\
\hline Article history: \\
Received Jun 1, 2019 \\
Revised Sep 2, 2019 \\
Accepted Sep 16, 2019
\end{tabular}

\section{Keywords:}

Demand response

Microgrid energy system

Robust framework

Uncertainty

\begin{abstract}
Microgrid energy systems are one of suitable solutions to the available problems in power systems such as energy losses, and resiliency issues. Local generation by these energy systems can reduce the role of the upstream network, which is a challenge in risky conditions. Also, uncertain behavior of electricity consumers and generating units can make the optimization problems sophisticated. So, uncertainty modeling seems to be necessary. In this paper, in order to model the uncertainty of generation of photovoltaic systems, a scenario-based model is used, while the robust optimization method is used to study the uncertainty of load. Moreover, the stochastic scheduling is performed to model the uncertain nature of renewable generation units. Time-of-use rates of demand response program (DRP) is also utilized to improve the system economic performance in different operating conditions. Studied problem is modeled using a mixed-integer linear programming (MILP). The general algebraic modeling system (GAMS) package is used to solve the proposed problem. A sample microgrid is studied and the results with DRP and without DRP are compared. It is shown that same robustness is achieved with a lower increase in the operation cost using DRP.
\end{abstract}

Copyright $@ 2020$ Institute of Advanced Engineering and Science. All rights reserved.

\section{Corresponding Author:}

Ali Farzamnia,

Faculty of Engineering,

Universiti Malaysia Sabah,

Kota Kinabalu, Sabah, Malaysia.

Email: ali-farzamnia@ieee.org

\section{INTRODUCTION}

Microgrid is a scaled-down power system that can benefit from local generation units to supply the energy demands. Optimal operation of microgrid is challenged by various factors including uncertainties, which can threaten the normal operation of microgrid energy systems [1-5]. Therefore, the robust operation of microgrid should be investigated.

A brief summary of studied papers about microgrid opera-tions is presented in the following: Implementing a two-stage adaptive robust optimization-based collaborative operation, the economic and collabo-rative operation of multi-microgrids has been evaluated as a unit commitment problem [6]. The total operation cost of multi-microgrids was reduced subject to uncertainty of so-lar power systems. In [7], optimal design of a hybrid electric power generation system for isolated zones has been inves-tigated and the total cost of power generation was mini-mized using particle swarm optimization. With the aim of calculating the sharing power of distributed energy re-sources with uncertainty and nonlinear load, the robust multi-objective control method has been employed in [8]. Optimal design and scheduling of power dispatch in a mi-crogrid has been achieved using a sliding time window op-timization modeling considering the unbalance of 
biomass-integrated renewable energy and energy storage [9]. In or-der to optimize the performance of energy management in the presence of renewable energy resources, a multi-objective generation scheduling model was developed un-der demand response program [10]. By implementing de-mand response program (DRP), the optimum components sizes in a microgrid was evaluated to decrease the cost of energy in [11]. With the aim of solving microgrids operation problem in the presence of electric vehicles, the robust op-timization has been implemented to reduce the operation cost of microgrid in [12]. In order to improve the characteris-tics of electric power distribution systems, conventional power generators and various types of renewable energy resources and electric vehicles have been used to improve the voltage profile and decrease the cost of power losses and energy [13]. Robust technique has been employed to control an isolated microgrid in the presence of high pene-tration of renewable energy sources [14]. Risk-based optimal operation of microgrid system is evaluated using robust optimization method in the presence of electric vehicles in [15]. Distribution system with multi-microgrid is optimally scheduled under uncertainty with robust optimization method in [16]. Similarly, robust optimization approach is used in [17] to solve uncertainty-based optimal energy transactions problem in multi-microgrid systems. In [18], a brief summary of studied papers about operation of photovoltaic based microgrids has been presented to investi-gate various combination methods of energy storage and electric vehicles with other energy resources. It is notewor-thy that optimal scheduling of microgrid systems have been studied under different types of demand response programs in several research papers [19-21] which repre-sents essential role of these programs in improvement of optimization results.

In this paper, the robust operation of microgrid energy system subject to uncertainties of load and renewable gen-eration unit is studied considering the time-of-use rates of demand response program. In order to model the uncer-tainty of generation of photovoltaic systems, a scenario-based model is used, while the robust optimization method is used to study the uncertainty of load. Combination of robust optimization method, which is a powerful strategy providing uncertain modeling method, with stochastic programming to model uncertainty ensures optimal reaction of operator against possible negative consequences of uncertainty. Furthermore, demand response can enhance effec-tiveness of these methods in uncertain handling.

It should be noted that the level of robustness depends on the policies taken by the operator of microgrids. According to each desired level of robustness, operation cost of mi-crogrid is increased. Therefore, robust optimization method can be used as a powerful uncertainty modelling tool to provide operation strategies for energy systems against different uncertainties. The studied problem is schematical-ly illustrated in Figure 1.

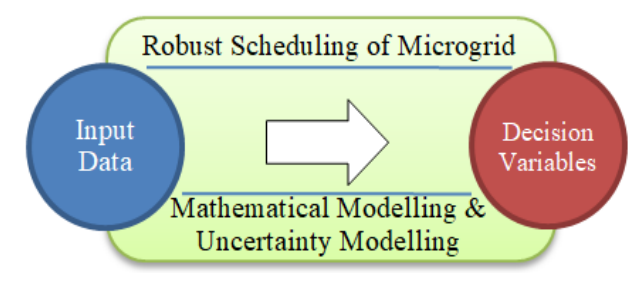

Figure 1. Studied problem

\subsection{Paper Structure}

The remaining parts of the paper are organized as: Robust operation of microgrid energy system is formulated in Sec-tion 2. The case studies and results of the proposed robust optimization scheme are presented in Section 3. Finally, the paper is concluded in Section 4.

\section{PROBLEM FORMULATION}

In this section, robust operation of microgrid energy system with load uncertainty is modelled, considering the demand response program.

\subsection{Objective Function} be minimized.

As the objective function, the cost of the purchased power from the upstream power grid (1) should

$$
\text { Min Cost }=\sum_{t=1}^{T} \lambda_{t} \times P_{t}^{n e t}
$$


In (1), Cost is the total operation cost of the microgrid; $\lambda_{t}$ and $P_{t}^{\text {net }}$ are the price and the amount of the power purchased from the upstream network.

\subsection{Constrains}

The power balance is formulated as

$$
P_{t}^{\text {Load }, D R P}+P_{t}^{E s s, c h}=P_{t}^{P V}+P_{t}^{n e t}+P_{t}^{E s s, d i s}
$$

where $P_{t}^{\text {Load,DRP }}$ is the load with DRP; $P_{t}^{E s s, c h}$ is the charging power of the battery energy storage systems (ESS); $P_{t}^{P V}$ is the power generated by the solar system; $P_{t}^{\text {net }}$ is the power from the upstream network; and $P^{E s s, d i s}$ is the discharging power of battery ESS.

The active power generated by the photovoltaic system is expressed as

$$
P_{t}^{P V}=A \times R_{t} \times \eta_{P V}
$$

where $A$ is the installation area; $R_{t}$ is the solar radiation; and $\eta_{P V}$ is the PV output efficiency.

The power imported from the upstream network is limited as

$$
P_{\min }^{n e t} \leq P_{t}^{n e t} \leq P_{\max }^{n e t}
$$

where $P_{\max }^{\text {net }}$ and $P_{\min }^{\text {net }}$ are the maximum and minimum allowable powers.

The stored energy in battery ESS depends on the state of charge of the battery at previous time step and the charging/discharging power at current time interval. The available energy is

$$
W_{t}^{E s s}=W_{t-1}^{E s s}+P_{t}^{E s s, c h} \times \eta^{c h}-P_{t}^{E s s, d i s} / \eta^{d i s}
$$

where $W_{t}^{E s s}$ is the stored energy level; $P_{t}^{E s s, c h}$ and $P_{t}^{E s s, d i s}$ are the charging and discharging powers; and $\eta^{c h}$ and $\eta^{\text {dis }}$ are the charging and discharging efficiencies of the battery ESS, respectively.

The stored energy of the battery ESS is limited as

$$
W_{\text {Min }}^{E s s} \leq W_{t}^{E s s} \leq W_{\text {Max }}^{\text {Ess }}
$$

where $W_{M a x}^{\text {Ess }}$ and $W_{M i n}^{\text {Ess }}$ are maximum and minimum energy of battery ESS, respectively.

The charging and discharging powers of battery ESS are limited by (7)-(8).

$$
\begin{aligned}
& \alpha_{t}^{d i s} \times P_{\min }^{E s s, d i s} \leq P_{t}^{E s s, d i s} \leq \alpha_{t}^{d i s} \times P_{\max }^{E s s, d i s} \\
& \alpha_{t}^{c h} \times P_{\min }^{E s s, c h} \leq P_{t}^{E s s, c h} \leq \alpha_{t}^{c h} \times P_{\max }^{E s s, c h}
\end{aligned}
$$

where $P_{\min }^{E s s, d i s}, P_{\max }^{E s s, d i s} P_{\min }^{E s s, c h}$ and $P_{\max }^{E s s, c h}$ are the minimum and maximum discharge and charging power limits of the battery ESS, respectively.

In (7) and (8), $\alpha_{t}^{\text {dis }}$ and $\alpha_{t}^{c h}$ are binary variables of discharging and charging of the battery ESS, which are limited as (9) since the battery ESS is either in charging or discharging.

$\alpha_{t}^{c h}+\alpha_{t}^{d i s} \leq 1$

The demand response program with the time-of-use method is modeled based on (10)-(13).

$$
P_{t}^{\text {Load }, D R P}=P_{t}^{\text {Load }}+p_{t}^{\text {shup }}-p_{t}^{\text {shdo }}
$$


where $P_{t}^{\text {Load }}$ is the base demand, $p_{t}^{\text {shup }}$ and $p_{t}^{\text {shdo }}$ are the increased and decreased values of the base load in DRP, which are limited as (11) and (12), respectively.

$$
\begin{aligned}
& 0 \leq p_{t}^{\text {shup }} \leq L P F^{\text {shup }} \times P_{t}^{\text {Load }} \times I_{t}^{\text {shup }} \\
& 0 \leq p_{t}^{\text {shdo }} \leq L P F^{\text {shdo }} \times P_{t}^{\text {Load }} \times I_{t}^{\text {shdo }}
\end{aligned}
$$

where $L P F^{\text {shup }}$ and $L P F^{\text {shdo }}$ are the limitation of the increase and decrease in load, and $I_{t}^{\text {shup }}$ and $I_{t}^{\text {shdo }}$ are binary variables to represent the increase of load in DRP.

The increased and decreased powers for DRP are balanced as:

$$
\sum_{t}^{T} p_{t}^{\text {shup }}=\sum_{t}^{T} p_{t}^{\text {shdo }}
$$

where $T$ is the scheduling horizon.

The robust optimization method and the different uncertainties are modelled as (14)-(20) to formulate the robust operation of microgrid energy system [22-23]. As shown, after implementing robust optimization, deviation of uncertain parameter are added to the objective function (14). (15) refers to the constants of base problem. As shown in (16)-(20) are used to model deviation of uncertain parameter. Then, by applying formulations presented in [22-23], the studied problem under uncertainty can be expressed as:

$$
\operatorname{Min}\left(\sum_{t=1}^{T} \lambda_{t} \times P_{t}^{n e t}+z_{0} \Gamma_{0}+\sum_{t=1}^{T} q_{o t}\right)
$$

s.t.

(2)-(13)

$$
z_{0}+q_{o t} \geq d_{t} y_{t}, \quad t=1, \ldots, T
$$

$$
q_{o t} \geq 0, \quad t=1, \ldots, T
$$

$y_{t} \geq 0, \quad t=1, \ldots, T$

$z_{0} \geq 0$

$y_{t} \geq P_{t}^{n e t}$

It should be noted that $z_{0}$ and $q_{o t}$ are dual variables of optimization problem. Duel variables are formed when dual form of an optimization problem is written using duality theorem. Also, $\Gamma_{0}$ is an integer defined to determine either uncertainty is considered or not. When uncertainty is considered, $\Gamma_{0}=1$ and otherwise $\Gamma_{0}=0$.

\section{NUMERICAL STUDIES}

Robust operation of microgrid energy system is evaluated considering the uncertainties and the DRP in this section. The input data used for simulations is presented in Subsection 3.1. The proposed robust optimization problem is solved and the results without and with DRP are given in Subsections 3.2 and 3.3 in the following, respectively. The studied test system is captured in Figure 2. 


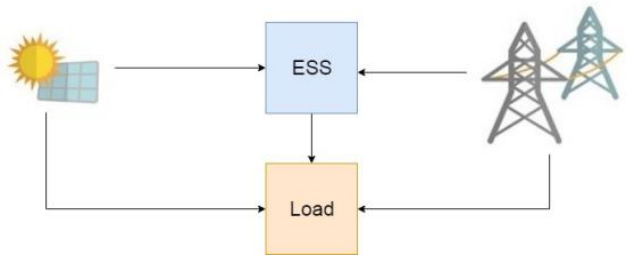

Figure 2. Test systems

\subsection{Input Data}

The hourly solar radiation is illustrated in Figure 3 [24]. The price of the power imported from the upstream network is illustrated in Figure 4 [25]. The energy demand of microgrid in different levels is illustrated in Figure 5 [25]. As illustrated, load is assumed to fluctuate between 0.7 and 1.3 of its base value. The technical data of battery ESS is provided in Table 1. It should be noted large number of scenarios are generated and then reduced for renewable units and the expected values are calculated to be used in the optimiza-tion process.

Table 1. ESS Characteristics

\begin{tabular}{lcccccccc}
\hline Parameter & $\mathrm{W}_{\min }^{\text {ess }}$ & $\mathrm{W}_{\max }^{\text {ess }}$ & $P_{\min }^{\text {ch }}$ & $P_{\max }^{\text {ch }}$ & $P_{\min }^{\text {dis }}$ & $P_{\max }^{\text {dis }}$ & $\eta_{t}^{\text {ch }}$ & $\eta_{t}^{\text {dis }}$ \\
\hline Value & $0 \mathrm{kWh}$ & $7.5 \mathrm{kWh}$ & $0 \mathrm{kWh}$ & $3 \mathrm{kWh}$ & $0 \mathrm{kWh}$ & $3 \mathrm{kWh}$ & $95 \%$ & $95 \%$ \\
\hline
\end{tabular}

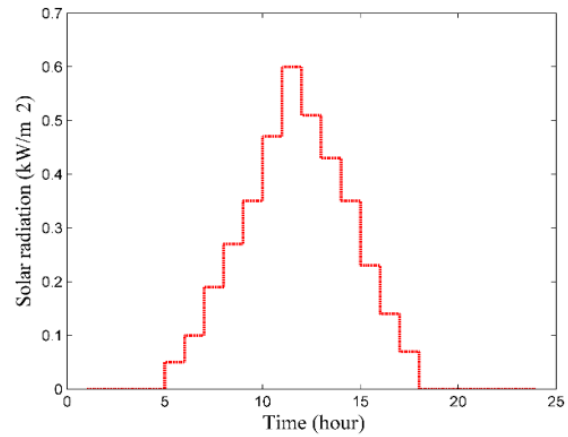

Figure 3. Solar radiation

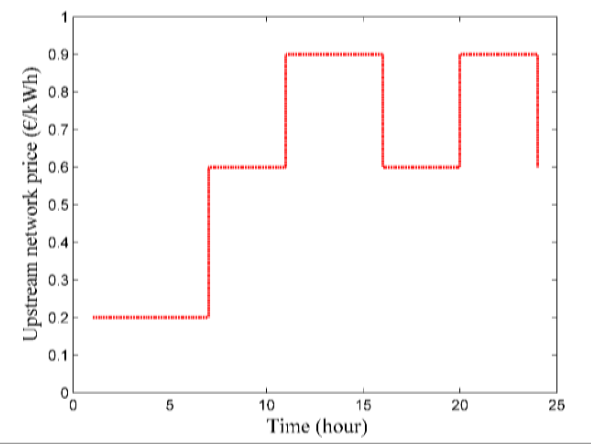

Figure 4. Upstream network price

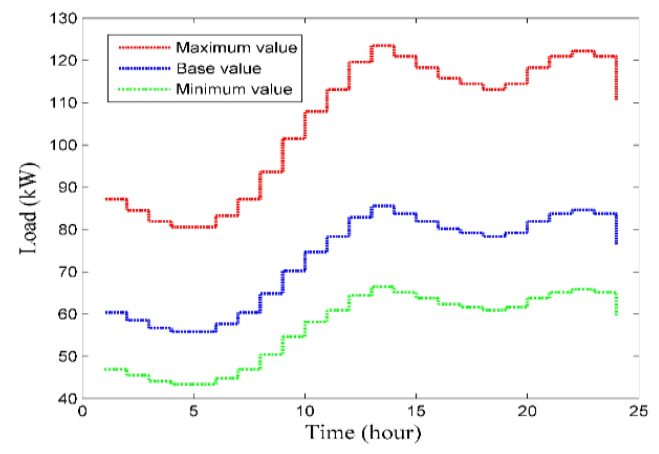

Figure 5. Upstream network price

\subsection{Results of Robust Energy Management without DRP}

In this section, results of the robust and stochastic scheduling of the microgrid energy system are presented. Calculating the expected values of solar radiation in each hour, the robust optimization method is applied to provide strategy for different levels of load uncertainty without DRP. The load is assumed to 
fluctuate between 0.7 and 1.3 of its base value. It should be noted that these values are selected random and can change based on the expectations of operator. Therefore, the most possible value of the load which is threatening the usual operation of the microgrid is 1.3 times the base value. Accordingly, the simulations are performed for several steps and the results are obtained. The total operation costs of microgrid against different levels of load uncertainty are presented in Table 2.

Table 2. Robust Cost without DRP

\begin{tabular}{lccccccc}
\hline Load uncertainty level (of base value) & 0.7 & 0.8 & 0.9 & 1.0 & 1.1 & 1.2 \\
\hline Operation cost of microgrid $(€)$ & 829.779 & 956.269 & 1082.759 & 1209.249 & 1335.739 & 1462.229 & 1588.719 \\
\hline
\end{tabular}

According to the results presented in Table 2, in order to become robust against $30 \%$ variation in load, the operation cost of microgrid energy system should be increased by 31.38 , to guarantee its normal operation against $30 \%$ additional load. On the other hand, when the load value is reduced to $70 \%$ of its base value, the operator of microgrid energy system can gain an economic profit up to $379.47 €$. In fact, due to reduction of load value up to $30 \%$, daily operation cost of microgrid energy system will decrease up to 31.38 $\%$. Therefore, by employing each one of the provided strategies by robust optimization method, the energy system can be resilient against different levels of load uncertainties. As a result, the imported power from the upstream network in different load uncertainty levels without DRP is obtained which is illustrated in Figure 6.

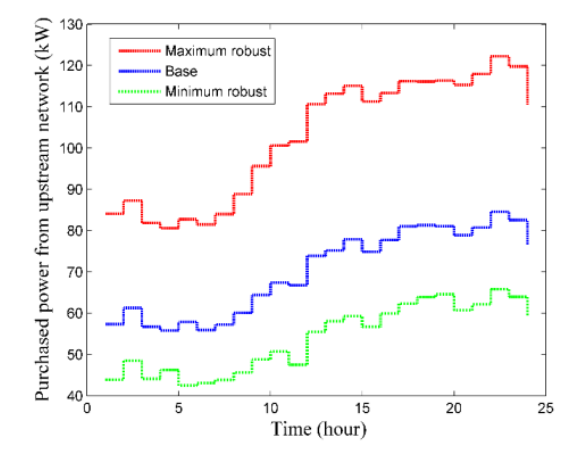

Figure 6. Upstream network power without DRP

In detail, when the uncertainty of load is not considered, the total power provided by the upstream network is $1881.753 \mathrm{~kW}$. When the load is in maximum level, total imported power from the upstream network is $2466.453 \mathrm{~kW}$. On the other hand, when the load is minimum, the total imported power is $1297.053 \mathrm{~kW}$.

\subsection{Results of Robust Energy Management with DRP}

Applying the time-of-use program of the DRP, the total operation costs against different levels of load uncertainty are obtained and presented in Table 3. It can be seen that the maximum value of load uncertainty can be handled by $5.05 \%$ less increase in operation cost under DRP which is economically beneficial for the microgrid operator. Moreover, by reduction of load value to $70 \%$ of its base value, the microgrid energy system can benefit $9.68 \%$ more reduction in cost utilizing the DRP. The power imported from the upstream network in this case is illustrated in Figure 7. As shown, the load is shifted from the peak periods to off-peak ones, and thus the power is purchased economically optimal to reduce the total operation cost as much as possible.

Table 3. Robust Cost with DRP

\begin{tabular}{lccccccc}
\hline Load uncertainty level (of base value) & 0.7 & 0.8 & 0.9 & 1.0 & 1.1 & 1.2 & 1.3 \\
\hline Operation cost of microgrid $(€)$ & 749.399 & 875.889 & 1002.379 & 1128.869 & 1255.359 & 1381.849 & 1508.339 \\
\hline
\end{tabular}




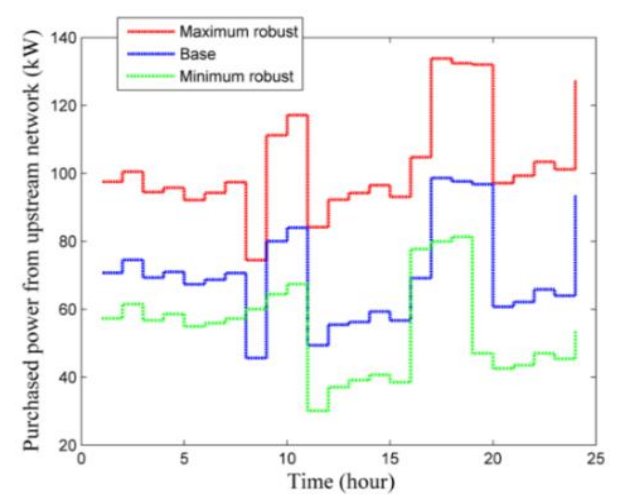

Figure 7. Upstream network power with robust scheduling and DRP

\subsection{Comparisons}

To approve the positive role of DRP on the uncertainty-based performance of the microgrid, simulation results are presented in Table 4, with and without taking the DRP into consideration.

Table 4. Comparison Results

\begin{tabular}{ccc}
\hline Load uncertainty level & \multicolumn{2}{c}{ Operation cost of microgrid $(€)$} \\
\cline { 2 - 3 } (fraction of base value) & Without DRP & With DRP \\
0.7 & 829.779 & 749.399 \\
0.8 & 956.269 & 875.889 \\
0.9 & 1082.759 & 1002.379 \\
1 & 1209.249 & 1128.869 \\
1.1 & 1335.739 & 1255.359 \\
1.2 & 1462.229 & 1381.849 \\
1.3 & 1588.719 & 1508.339 \\
\hline
\end{tabular}

\section{CONCLUSION}

In this paper, robust and stochastic operation of microgrid energy system is studied considering the uncertainties in load and renewable power of PV system. A large number of scenarios are generated first and then reduced using the fast-forward scenario reduction algorithm. Consequently, the expected values of uncertain parameters related to renewable power of PV system are calculated. The robust scheduling of the microgrid energy management is formulated as a mixed-integer linear programming (MILP) optimization problem. According to the results, robust scheduling of microgrid energy system in the worst condition is obtained against $30 \%$ higher load level compared to the base load, while daily operation cost of microgrid is increased by $31.38 \%$ without DRP. However, the same robustness can be achieved with a lower increase in the operation cost using the DRP, which is more economically beneficial for the microgrid operator. The proposed robust optimization method can be used as a powerful uncertainty modelling tool to provide operation strategies for energy systems against different uncertainties.

\section{REFERENCES}

[1] Jadidbonab M, Babaei E, Mohammadi-ivatloo B. "CVaR-constrained Scheduling Strategy for Smart Multi Carrier Energy Hub Considering Demand Response and Compressed Air Energy Storage”. Energy. 2019 Feb 11.B.

[2] Fazlalipour P, Ehsan M, Mohammadi-Ivatloo B. "Risk-aware stochastic bidding strategy of renewable micro-grids in day-ahead and real-time markets". Energy. 2019 Mar 15;171:689-700.B.

[3] Dolatabadi A, Mohammadi-Ivatloo B. "Stochastic risk-constrained scheduling of smart energy hub in the presence of wind power and demand response". Applied Thermal Engineering. 2017 Aug 1;123:40-9.

[4] Nazari-Heris M, Abapour S, Mohammadi-ivatloo B. "Robust Optimization Method for Obtaining Optimal Scheduling of Active Distribution Systems Considering Uncertain Power Market Price". InRobust Optimal Planning and Operation of Electrical Energy Systems, 2019, pp. 293-308. Springer, Cham.

[5] Nazari-Heris M, Abapour S, Mohammadi-ivatloo B. "Robust Optimization Method for Obtaining Optimal Scheduling of Active Distribution Systems Considering Uncertain Power Market Price". InRobust Optimal Planning and Operation of Electrical Energy Systems 2019, pp. 293-308. Springer, Cham.

[6] B. Zhang, Q. Li, L. Wang, and W. Feng, "Robust optimization for energy transactions in multi-microgrids under uncertainty," Applied Energy, vol. 217, pp. 346-360, 2018. 
[7] A. L. G. Noguera, L. S. M. Castellanos, E. E. S. Lora, and V. R. M. Cobas, "Optimum design of a hybrid dieselORC/photovoltaic system using PSO: Case study for the city of Cujubim, Brazil," Energy, vol. 142, pp. 33-45, 2018.

[8] S. Gholami, S. Saha, and M. Aldeen, "Robust multiobjective control method for power sharing among distributed energy resources in islanded microgrids with unbalanced and nonlinear loads," International Journal of Electrical Power \& Energy Systems, vol. 94, pp. 321-338, 2018.

[9] Y. Zheng, B. M. Jenkins, K. Kornbluth, and C. Træholt, "Optimization under uncertainty of a biomass-integrated renewable energy microgrid with energy storage," Renewable Energy, vol. 123, pp. 204-217, 2018.

[10] G. Aghajani, H. Shayanfar, and H. Shayeghi, "Presenting a multi-objective generation scheduling model for pricing demand response rate in micro-grid energy management," Energy Conversion and Management, vol. 106, pp. 308-321, 2015.

[11] M. H. Amrollahi and S. M. T. Bathaee, "Techno-economic optimization of hybrid photovoltaic/wind generation together with energy storage system in a stand-alone micro-grid subjected to demand response," Applied Energy, vol. 202, pp. 66-77, 2017.

[12] S. Bahramara and H. Golpîra, "Robust optimization of micro-grids operation problem in the presence of electric vehicles," Sustainable Cities and Society, vol. 37, pp. 388-395, 2018.

[13] H. Fathabadi, "Utilization of electric vehicles and renewable energy sources used as distributed generators for improving characteristics of electric power distribution systems," Energy, vol. 90, pp. 1100-1110, 2015.

[14] Nazari-Heris, M., Madadi, S., \& Mohammadi-Ivatloo, B. (2018). "Optimal management of hydrothermal-based micro-grids employing robust optimization method. In Classical and recent aspects of power system optimization, pp. 407-420.

[15] Bahramara S, Golpîra H. "Robust optimization of micro-grids operation problem in the presence of electric vehicles". Sustainable cities and society. $2018 \mathrm{Feb}$ 28;37:388-95.

[16] Liu Y, Guo L, Wang C. "A robust operation-based scheduling optimization for smart distribution networks with multi-microgrids". Applied Energy. 2018 Oct 15;228:130-40.

[17] Zhang B, Li Q, Wang L, Feng W. "Robust optimization for energy transactions in multi-microgrids under uncertainty". Applied Energy. 2018 May 1;217:346-60.

[18] H. Fathabadi, "Novel battery/photovoltaic hybrid power source for plug-in hybrid electric vehicles," Solar Energy, vol. 159, pp. 243-250, 2018.

[19] Shams MH, Shahabi M, Khodayar ME. "Stochastic day-ahead scheduling of multiple energy Carrier microgrids with demand response". Energy. 2018 Jul 15;155:326-38.

[20] Li B, Roche R, Paire D, Miraoui A. "A price decision approach for multiple multi-energy-supply microgrids considering demand response". Energy. 2018 Nov 2.

[21] Ghasemi A, Enayatzare M. "Optimal energy management of a renewable-based isolated microgrid with pumpedstorage unit and demand response". Renewable Energy. 2018 Aug 1;123:460-74.

[22] D. Bertsimas and M. Sim, "Robust discrete optimization and network flows," Mathematical programming, vol. 98, no. $1-3$, pp. 49-71, 2003.

[23] K. Knezović, A. Soroudi, A. Keane, and M. Marinelli, "Robust multi-objective PQ scheduling for electric vehicles in flexible unbalanced distribution grids," IET Generation, Transmission \& Distribution, vol. 11, no. 16, pp. 4031-4040, 2017.

[24] H. Ren, Q. Wu, W. Gao, and W. Zhou, "Optimal operation of a grid-connected hybrid PV/fuel cell/battery energy system for residential applications," Energy, vol. 113, pp. 702-712, 2016.

[25] M. Elsied, A. Oukaour, H. Gualous, and O. A. L. Brutto, "Optimal economic and environment operation of microgrid power systems," Energy conversion and management, vol. 122, pp. 182-194, 2016.

\section{BIOGRAPHIES OF AUTHORS}
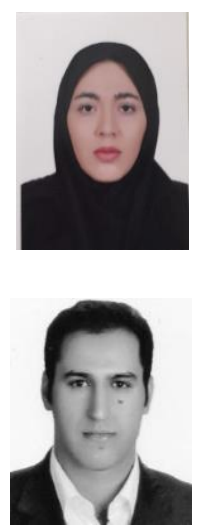

Sahar Seyyedeh-Barhagh received the B.Sc. Azarbaijan Shahid Madani University and M.Sc. degrees from University of Tabriz, Tabriz, Iran, in 2015 and 2018, respectively. Her research areas include electric vehicles, distribution systems and power system economics.

Amin Mohammadpour Shotorbani received his B.Sc. degree from the University of Tabriz, Tabriz, Iran, in 2009, the M.Sc. degree from Azarbaijan Shahid Madani University, Tabriz, Iran, in 2012, and the Ph.D. degree from the University of Tabriz, Tabriz, Iran, in 2017, all in electrical engineering. From Jan. 2016 to June 2016, he was a Visiting Researcher with the University of British Columbia, Kelowna, BC, Canada. From Sept. 2017 to June 2018, he was a Postdoctoral Research Fellow with the University of Tabriz. Since July 2018, he has been a Postdoctoral Research Fellow with the University of British Columbia, Kelowna, BC, Canada. His main area of research is nonlinear control methods, operation of modern power and energy management systems, microgrids, and HVDC and FACTS. 

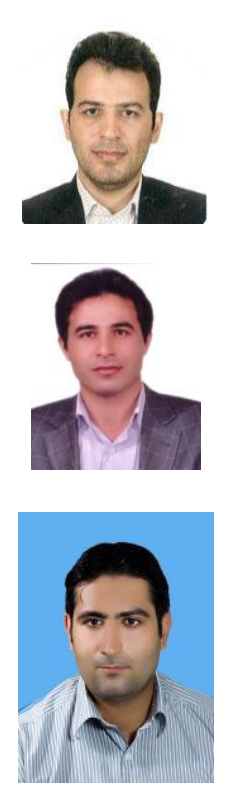

Behnam Mohammadi-Ivatloo received his B.Sc. degree in Electrical Engineering from University of Tabriz, Tabriz, Iran in 2006, the M.Sc. and PhD degree from the Sharif University of Technology, Tehran, Iran in 2008 and 2012, respectively. He was a research associate at the University of Calgary from June 2011 to March 2012. He is currently an Associate Professor in the Faculty of Electrical and Computer Engineering, University of Tabriz, Tabriz, Iran. His main area of research is operation of modern power systems

Kazem Zare received the B.Sc. and M.Sc. degrees in electrical engineering from University of Tabriz, Tabriz, Iran, in 2000 and 2003, respectively, and Ph.D. degree from Tarbiat Modares University, Tehran, Iran, in 2009. Currently, he is an Associate Professor of the Faculty of Electrical and Computer Engineering, University of Tabriz. His research areas include power system economics, distribution networks, microgrid and energy management.

Ali Farzamnia received his B. Eng in Electrical Engineering (Telecommunication Engineering) from Islamic Azad University of Urmia IRAN 2005. He got M.Sc in Electrical Engineering (Telecommunication Engineering) from university of Tabriz in 2008. He obtained $\mathrm{PhD}$ in Electrical Engineering (Telecommunication Engineering) from Universiti Teknologi Malaysia (UTM) at 2014. Since 2014, He is appointed as senior lecturer in the Electrical and Electronic Engineering program, Faculty of Engineering at Universiti Malaysia Sabah (UMS). He is senior member of IEEE and member of IET. Dr Ali's research interests are Wireless Communication, Signal Processing, Network coding and Information Theory. 